\title{
$\alpha$-Lactalbumin-oleic acid complex kills tumor cells by inducing excess energy metabolism but inhibiting mRNA expression of the related enzymes
}

\author{
B. Fang, ${ }^{* 1}$ M. Zhang, † K. S. Ge, $\ddagger$ H. Z. Xing, ${ }^{*}$ and F. Z. Ren* $¥$ \\ *Beijing Advanced Innovation Center for Food Nutrition and Human Health, College of Food Science and Nutritional Engineering, China \\ Agricultural University, Beijing 100083, China \\ †School of Food Science and Chemical Engineering, Beijing Technology and Business University, Beijing 100048, China \\ ¥Key Laboratory of Functional Dairy, co-constructed by Ministry of Education and Beijing Government, \\ and Beijing Laboratory of Food Quality and Safety, China Agricultural University, Beijing 100083, China
}

\section{ABSTRACT}

Previous studies have demonstrated that the anti-tumor $\alpha$-lactalbumin-oleic acid complex ( $\alpha$-LA-OA) may target the glycolysis of tumor cells. However, few data are available regarding the effects of $\alpha-\mathrm{LA}-\mathrm{OA}$ on energy metabolism. In this study, we measured glycolysis and mitochondrial functions in HeLa cells in response to $\alpha$-LA-OA using the XF flux analyzer (Seahorse Bioscience, North Billerica, MA). The gene expression of enzymes involved in glycolysis, tricarboxylic acid cycle, electron transfer chain, and ATP synthesis were also evaluated. Our results show that $\alpha$-LA-OA significantly enhanced the basal glycolysis and glycolytic capacity. Mitochondrial oxidative phosphorylation, including the basal respiration, maximal respiration, spare respiratory capacity and ATP production were also improved in response to $\alpha-\mathrm{LA}-\mathrm{OA}$. The enhanced mitochondrial functions maybe partly due to the increased capacity of utilizing fatty acids and glutamine as the substrate. However, the gene expressions of pyruvate kinase M2, lactate dehydrogenase $\mathrm{A}$, aconitate hydratase, and isocitrate dehydrogenase 1 were inhibited, suggesting an insufficient ability for the glycolysis process and the tricarboxylic acid cycle. The increased expression of acetyl-coenzyme A acyltransferase 2, a central enzyme involved in the $\beta$-oxidation of fatty acids, would enhance the unbalance due to the decreased expression of electron transfer flavoprotein $\beta$ subunit, which acts as the electron acceptor. These results indicated that $\alpha$-LA-OA may induce oxidative stress due to conditions in which the ATP production is exceeding the energy demand. Our results may help clarify the mechanism of apoptosis induced by reactive oxygen species and mitochondrial destruction.

Received August 22, 2017.

Accepted November 18, 2017.

${ }^{1}$ Corresponding author: bingfang@cau.edu.cn or 49631263@qq.com
Key words: glycolysis, tricarboxylic acid cycle, mitochondrial oxidative phosphorylation, extracellular acidification rate, oxygen consumption rate

\section{INTRODUCTION}

The selective tumoricidal activity of $\alpha$-lactalbuminoleic acid complex ( $\boldsymbol{\alpha}-\mathbf{L A} \mathbf{A}-\mathbf{O A})$ has been confirmed in murine models of glioblastoma xenograft and bladder cancer (Fischer et al., 2004; Mossberg et al., 2010) and in clinical studies of skin papillomas and bladder cancers (Gustafsson et al., 2004; Mossberg et al., 2007). To investigate the conserved survival mechanisms that are targeted by $\alpha-\mathrm{LA}-\mathrm{OA}$, several screening techniques, such as metabolomic technology (Storm et al., 2011) and the isobaric tags for relative and absolute quantitation method (Fang et al., 2016), have been used to identify genes or proteins targeted by $\alpha$-LA-OA.

In contrast to healthy cells, tumor cells must support continuous cell growth and proliferation and thus exhibit high rates of aerobic glycolysis, which converts pyruvate to lactate acid instead of acetyl-CoA, a necessary component of the tricarboxylic acid (TCA) cycle, even in the presence of oxygen (known as the Warburg effect; Hsu and Sabatini, 2008). A previous study showed that depletion of glucose or addition of 2-deoxyglucose (2-DG), a glycolysis inhibitor, increases the sensitivity of tumor cells to HAMLET, a similar complex of $\alpha$-LA with OA (Storm et al., 2011), indicating that $\alpha$-LA-OA may target the energy metabolism of tumor cells. The authors found that the sensitivity of tumor cells to $\alpha$-LA-OA was dependent on c-Myc and glycolysis toward the pentose phosphate pathway by inhibiting hexokinase and pyruvate kinase isozymes M2 was increased by $\alpha$-LA-OA (Storm et al., 2011). Furthermore, our previous proteomics study reported that $\alpha$-LA-OA increased the expression of poly (ADP-ribose) polymerase 1 (PARP-1), which caused cell death by inhibition of hexokinase activity, leading to defects in glycolysis (Andrabi et al., 2014). Together 
these studies indicate that $\alpha-\mathrm{LA}-\mathrm{OA}$ alters cancer cell metabolism and that it may disrupt glycolysis, which is altered in cancer cells as discussed above. However, in contrast with these results of decreased glycolysis by $\alpha$-LA-OA, our proteomics results also demonstrated that $\alpha$-LA-OA increased the expression of several proteins involved in carbohydrate and energy metabolism, such as fructose-bisphosphate aldolase A, pyruvate kinase isozymes M1/M2, succinate dehydrogenase, electron transfer flavoprotein, and ATP synthase (Fang et al., 2016), indicating an enhanced glycolytic capacity and energy metabolism.

To clarify the energy metabolism changes induced by $\alpha$-LA-OA, here we used the XF flux analyzer to evaluate glycolysis and mitochondrial function in HeLa cells. This study may help clarify the effects of $\alpha$-LA-OA on the energy metabolism of tumor cells and may help identify some potential targets for tumor therapy.

\section{MATERIALS AND METHODS}

\section{Materials}

Bovine $\alpha$-LA ( $\geq 85 \%$ purity), oleic acid (OA, C18: $1: 9$ cis, $\geq 99.0 \%$ purity, cell culture tested), glucose, L-glutamine, and pyruvate were obtained from SigmaAldrich Chemical Co. (St. Louis, MO). HyClone Dulbecco's modified Eagle's medium (DMEM) containing a high level of glucose was purchased from GE Healthcare (Chicago, IL). Fetal bovine serum was purchased from Gibco (Thermo Fisher Scientific, Carlsbad, CA). All other chemicals used were of analytical grade.

\section{a-LA-OA Preparation}

The $\alpha$-LA-OA was prepared by heat treatment according to previously published methods (Fang et al., 2014), with modifications. We dissolved $\alpha$-LA in phosphate buffer solution $\left(\mathrm{Na}_{2} \mathrm{HPO}_{4}\right.$ and $\mathrm{NaH}_{2} \mathrm{PO}_{4} 10 \mathrm{mM}$, $\mathrm{pH}$ 8.0) to a final concentration of $1 \mathrm{~m} M$. Next, OA was directly added to the solution at 30 molar equivalents (OA:protein). The mixture was vortexed for $10 \mathrm{~s}$ and then incubated at $45^{\circ} \mathrm{C}$ in a water bath for $10 \mathrm{~min}$. The sample was centrifuged at $4^{\circ} \mathrm{C}, 12,000 \times g$ for $15 \mathrm{~min}$ to remove the unbound OA. The $\alpha$-LA solution treated as above but without addition of OA was used as the control $\alpha$-LA. Phosphate buffer added with same dose of OA with $\alpha-\mathrm{LA}-\mathrm{OA}$ and treated as above was used as control buffer.

\section{Cell Culture and Cell Viability Assay}

Human cervical carcinoma HeLa cells (Cell Bank of Chinese Academy of Science, Shanghai, China) were cultured in DMEM supplemented with $10 \%$ FBS in an incubator with $5 \% \mathrm{CO}_{2}$ at $37^{\circ} \mathrm{C}$.

For the viability assays, HeLa cells were seeded in 96well plates at a density of $1 \times 10^{4}$ cells/well and grown for $24 \mathrm{~h}$. The medium was removed and then replaced with fresh medium containing $\alpha$-LA-OA, the control $\alpha$-LA at final concentrations of 20,40,60, 80, or 100 $\mu M$. The same volume of control buffer corresponded to $20,40,60,80$, or $100 \mu M$ of $\alpha$-LA-OA was added into medium and taken as the control treatments. After $24 \mathrm{~h}$ incubation at $37^{\circ} \mathrm{C}$, cell viability was detected by the cell counting kit-8 (CCK-8, Dojindo Laboratories, Kumamoto, Japan). Next, $10 \mu \mathrm{L}$ of CCK-8 solution was added to each well, and the 96-well plate was incubated at $37^{\circ} \mathrm{C}$ for $1 \mathrm{~h}$. The optical density (OD) value for each well was read at $450 \mathrm{~nm}$ on a microplate reader (Infinite 200 PRO, Tecan, Zurich, Switzerland) to determine the cell viability. Wells did not contain cells and those treated as above were taken as blank wells. The assay was repeated 6 times. Cell viability was calculated using the following formula:

cell viability $(\%)=\frac{\mathrm{OD}(\text { experiment })-\mathrm{OD}(\text { blank })}{\mathrm{OD}(\text { control buffer })-\mathrm{OD}(\text { blank })} \times 100$.

\section{Glucose Uptake}

Cells were plated in 96-well plates at $1 \times 10^{4}$ cells per well and cultured for $24 \mathrm{~h}$. Next, $\alpha$-LA-OA or control $\alpha$-LA was added at various concentrations and cells were incubated for another $24 \mathrm{~h}$. Extracellular glucose levels were determined by the glucose oxidase method using a glucose determination kit (Applygen Technologies Inc., Beijing, China; Jiang et al., 2016). The glucose uptake of cells was subtracted the extracellular glucose levels from the glucose levels in the media.

\section{Measurement of Glycolysis as Extracellular Acidification Rate}

Cells were plated in Seahorse XF24 well plates (Seahorse Bioscience, North Billerica, MA) at $2 \times 10^{4}$ cells/ well and incubated for $12 \mathrm{~h}$ until confluent. After 24-h treatment of $\alpha$-LA-OA or control $\alpha$-LA, the culture medium was replaced with glucose-free XF24 Seahorse medium containing $1 \mathrm{~m} M$ L-glutamine. Glycolytic flux (basal glycolysis, glycolytic capacity, and glycolytic reserve) in HeLa cells as assessed by extracellular acidification rate (ECAR) was measured by the sequential addition of glucose $(10 \mathrm{mM})$, oligomycin $(1 \mu M)$, and 2-DG $(50 \mathrm{mM})$ in an XF24 Extracellular Flux Analyzer according to the manual of XF glycolysis stress test kit (no. 103020-100, Seahorse Bioscience). The ECAR 
values were normalized to the cell counts in each well. Data were presented as the percentage of change compared with control.

The basal glycolysis, glycolytic capacity, glycolytic reserve, and nonglycolytic acidification values were calculated using the following formulas:

$$
\text { Basal glycolysis }=\mathrm{ECAR}_{\text {point II }}-\mathrm{ECAR}_{\text {point I }} \text {; }
$$$$
\text { Glycolytic capacity }=\mathrm{ECAR}_{\text {point III }}-\mathrm{ECAR}_{\text {point II }} \text {; }
$$

Glycolytic reserve = glycolytic capacity - basal glycolysis;

$$
\text { Nonglycolytic acidification }=\mathrm{ECAR}_{\text {point } \mathrm{I}} \text {. }
$$

\section{Measurements of Mitochondrial Functions as Oxygen Consumption Rate}

Mitochondrial oxygen consumption rate (OCR) in HeLa cells was measured according to the manual of the XF Cell Mito Stress Test Kit (No. 103150-100, Seahorse Bioscience). Briefly, cells were plated in Seahorse XF24 well plates at $2 \times 10^{4}$ cells/well and incubated $12 \mathrm{~h}$ until confluent. After $24 \mathrm{~h}$ of treatment of $\alpha$-LAOA or control $\alpha$-LA, the culture medium was replaced with XF24 DMEM containing $10 \mathrm{~m} M$ glucose with 2 $\mathrm{m} M$ L-glutamine and $1 \mathrm{~m} M$ pyruvate. The OCR were measured at basal glucose levels as well as after $1 \mathrm{~h}$ incubation with drugs disrupting the respiratory chain in a $\mathrm{CO}_{2}$-free incubator at $37^{\circ} \mathrm{C}$; drug treatments included oligomycin (ATP synthase/complex V inhibitor, $2 \mu M$ ) and carbonyl cyanide p-trifluoromethoxyphenylhydrazone (inner mitochondrial membrane inhibitor, $1 \mu M$ ). Finally, the mitochondrial respiration was blocked by rotenone (mitochondrial complex I inhibitor, $0.5 \mu M$ ) and antimycin A (mitochondrial complex III inhibitor, $0.5 \mu M$ ). The OCR were normalized to the cell counts in each well.

The nonmitochondrial respiration, basal respiration, maximal respiration, proton leak, ATP production, and spare respiratory capacity values were calculated using the following formulas:

$$
\begin{gathered}
\text { Nonmitochondrial respiration }=\mathrm{OCR}_{\text {pointIV }} ; \\
\text { Basal respiration }=\mathrm{OCR}_{\text {point I }}-\mathrm{OCR}_{\text {point IV }} ; \\
\text { Maximal respiration }=\mathrm{OCR}_{\text {point III }}-\mathrm{OCR}_{\text {point IV }} ; \\
\text { Proton leak }=\mathrm{OCR}_{\text {point II }}-\mathrm{OCR}_{\text {point IV }}
\end{gathered}
$$

$$
\mathrm{ATP} \text { production }=\mathrm{OCR}_{\text {point II }}-\mathrm{OCR}_{\text {point I }} \text {; }
$$

Spare respiration capacity $=\mathrm{OCR}_{\text {point III }}-\mathrm{OCR}_{\text {point I }}$.

\section{Measurements of Mitochondrial Substrate Utilization as OCR}

Regulation of substrate utilization in response to $\alpha$-LA-OA was measured according to the protocol of the XF Mito Fuel Flex Test Kit (no. 103260-100, Seahorse Bioscience). Briefly, cells were plated in Seahorse XF24 well plates at $2 \times 10^{4}$ cells/well and incubated $12 \mathrm{~h}$ until confluent. After 24-h treatment of $\alpha$-LAOA or control $\alpha$-LA, the culture medium was replaced with XF24 DMEM containing $10 \mathrm{~m} M$ glucose with 2 $\mathrm{m} M$ L-glutamine and $1 \mathrm{~m} M$ pyruvate. The OCR were measured at basal glucose levels as well as with drugs inhibiting the utilization of the substrate after $1 \mathrm{~h}$ incubation in a $\mathrm{CO}_{2}$-free incubator at $37^{\circ} \mathrm{C}$. The dependency on glucose, glutamine, and long-chain fatty acids in HeLa cells was assessed by OCR changes with the addition of the specific inhibitors UK-5099 $(2 \mu M)$, bis2-(5-phenylacetamido-1,3,4-thiadiazol-2-yl)ethyl sulfide (BPTES; $3 \mu M)$, or etomoxir $(4 \mu M)$, respectively. The capacity for use of the 3 substrates was assessed by OCR changes with the addition of the specific inhibitor followed by the mixture of the other 2 inhibitors. The OCR were normalized to the cell counts in each well.

The dependency and capacity of each fuel were calculated using the following formulas:

Dependency $(\%)=$

OCR (the first injection) - OCR (the second injection)

OCR (the first injection) - OCR (the last point) $\times 100$;

$$
\text { Capacity }(\%)=100-\text { Dependency }(\%) \text {. }
$$

\section{Enzyme Gene Expression by Quantitative Real-Time PCR}

Total RNA was isolated using TRIzol reagent (Invitrogen, Carlsbad, CA). Complementary DNA was prepared from $800 \mathrm{ng}$ of RNA using the PrimeScript RT Master Mix (TaKaRa, Shiga, Japan) and diluted to a final volume of $30 \mu \mathrm{L}$. Diluted cDNA $(1 \mu \mathrm{L})$ was used in a $10-\mu \mathrm{L}$ PCR reaction with SYBR Green Master Mix (Applied Biosystems). The PCR reactions were run in triplicate for each sample and quantified using the Roche LightCycler 96 System (Roche). The relative expression of the mRNA determined after each mea- 

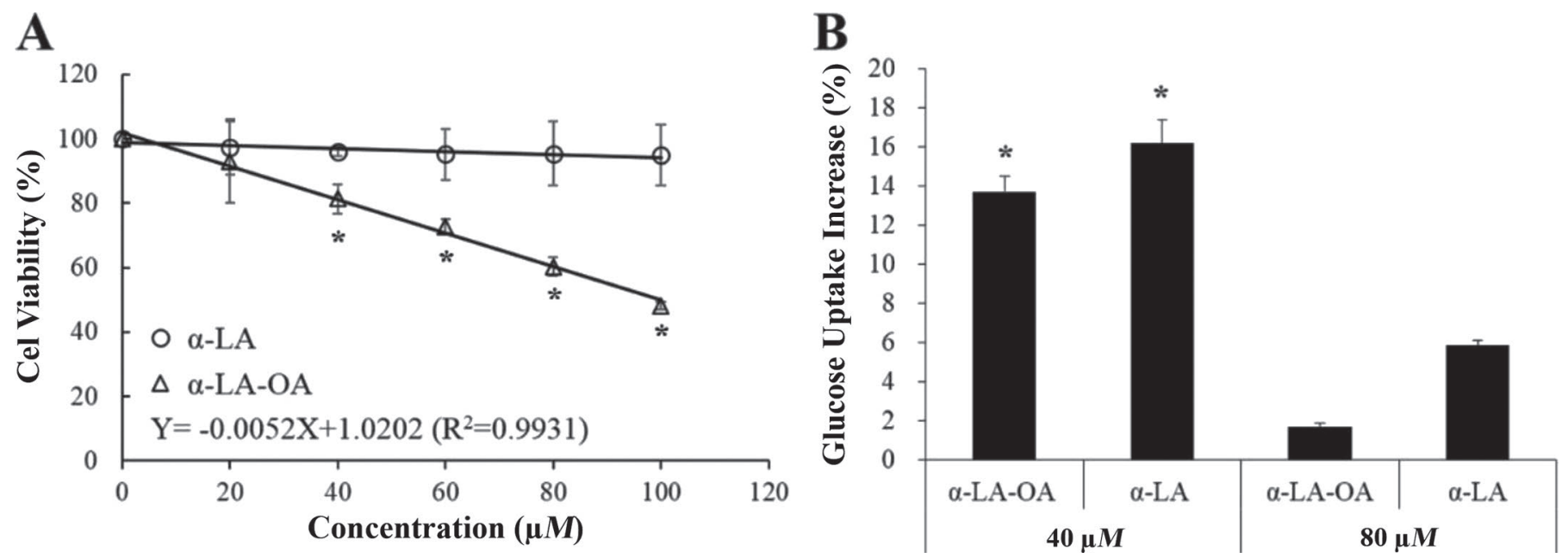

Figure 1. Cell viability (A) and glucose uptake (B) after 24-h treatment of HeLa cells with various concentrations of $\alpha$-lactalbumin-oleic acid complex ( $\alpha$-LA-OA; triangle) or control $\alpha$-LA (circle) compared with the control buffer. The data with error bars are expressed as mean \pm SD $(\mathrm{n}=6) .{ }^{*}$ indicates a significant difference at a CI of $95 \%$.

surement was normalized with the $18 \mathrm{~S}$ rRNA level as the internal control using the $2^{-\Delta \Delta \mathrm{CT}}$ method. All primers were synthesized by Invitrogen (Shanghai). The primers are listed in Supplemental Table S1 (https:// doi.org/10.3168/jds.2017-13731).

\section{Statistical Analysis}

Values are expressed as means \pm standard deviation of at least 3 independent experiments. Data were analyzed with SPSS statistical software (version 18.0) using one-way ANOVA followed by the Student $t$-test and Duncan's test, with the threshold for significance as $P<0.05$.

\section{RESULTS AND DISCUSSION}

\section{Anti-Tumor Activity and Glucose Uptake}

The cell viability and glucose uptake results of HeLa cells treated with $\alpha$-LA-OA compared with the control $\alpha-\mathrm{LA}$ are shown in Figure 1. According to the concentration-viability (X-Y) curves, the quantitative equation for each complex was $\mathrm{Y}=-0.0052 \mathrm{X}+1.0202$ $\left(\mathrm{R}^{2}=0.9931\right)$ and the half lethal doses $\left(\mathrm{LD}_{50}\right)$ of $\alpha$-LAOA was $100 \mu M$ (Figure 1A). No anti-tumor activity was observed for the control $\alpha$-LA at all the tested concentrations. Our previous study reported that OA could kill tumor cells in a dose-dependent way, and the actual molecular ratios of OA in $\alpha-\mathrm{LA}-\mathrm{OA}$ was 1 (Fang et al., 2014). Therefore, it can be speculated that the decrease in cell viability when increasing the dose of Q-LA-OA was caused by increased amounts of bound OA in the complex.
At the concentration of $40 \mu M$, which induced a significant decrease in cell viability, $\alpha$-LA-OA enhanced the total glucose uptake of HeLa cells compared with buffer treated cells; similar results were observed with $\alpha$-LA $(P<0.05$, Figure 1B). This was consistent with the increased expression of proteins involved in energy metabolism after a 24 -h treatment of $40 \mu M \alpha$-LA-OA (Fang et al., 2016). However, at higher concentrations of $\alpha$-LA-OA $(80 \mu M)$, the glucose uptake decreased to levels similar to those of the control $(P>0.05)$, indicating a possible feedback of $\alpha$-LA-OA on energy metabolism. As these measurements only evaluated the amount of glucose that entered cells, we next determined the actual metabolism of glucose in response to $\alpha$-LA-OA by examining the effect on glycolysis and mitochondrial function.

\section{a-LA-OA Enhanced the Aerobic Glycolytic Potential and Mitochondrial Oxidative Phosphorylation}

To determine whether $\alpha$-LA-OA influenced glycolysis, we performed assays to measure ECAR (Figure 2). Cells were first starved for $1 \mathrm{~h}$ and then a saturating concentration of glucose $(10 \mathrm{mM})$ was added (Figure $2 \mathrm{~A}$, point I). This glucose was catabolized through the glycolytic pathway to pyruvate and then converted to lactate in the cytoplasm, or produced ATP, $\mathrm{CO}_{2}$, and water in the mitochondria, causing a rapid increase in ECAR. This glucose-induced response is considered the rate of glycolysis under basal conditions (basal glycolysis). Notably, the basal glycolysis of HeLa cells to $\alpha$-LA-OA was increased compared with $\alpha$-LA and control (Figure 2B, $P<0.05$ ). Next, oligomycin was 

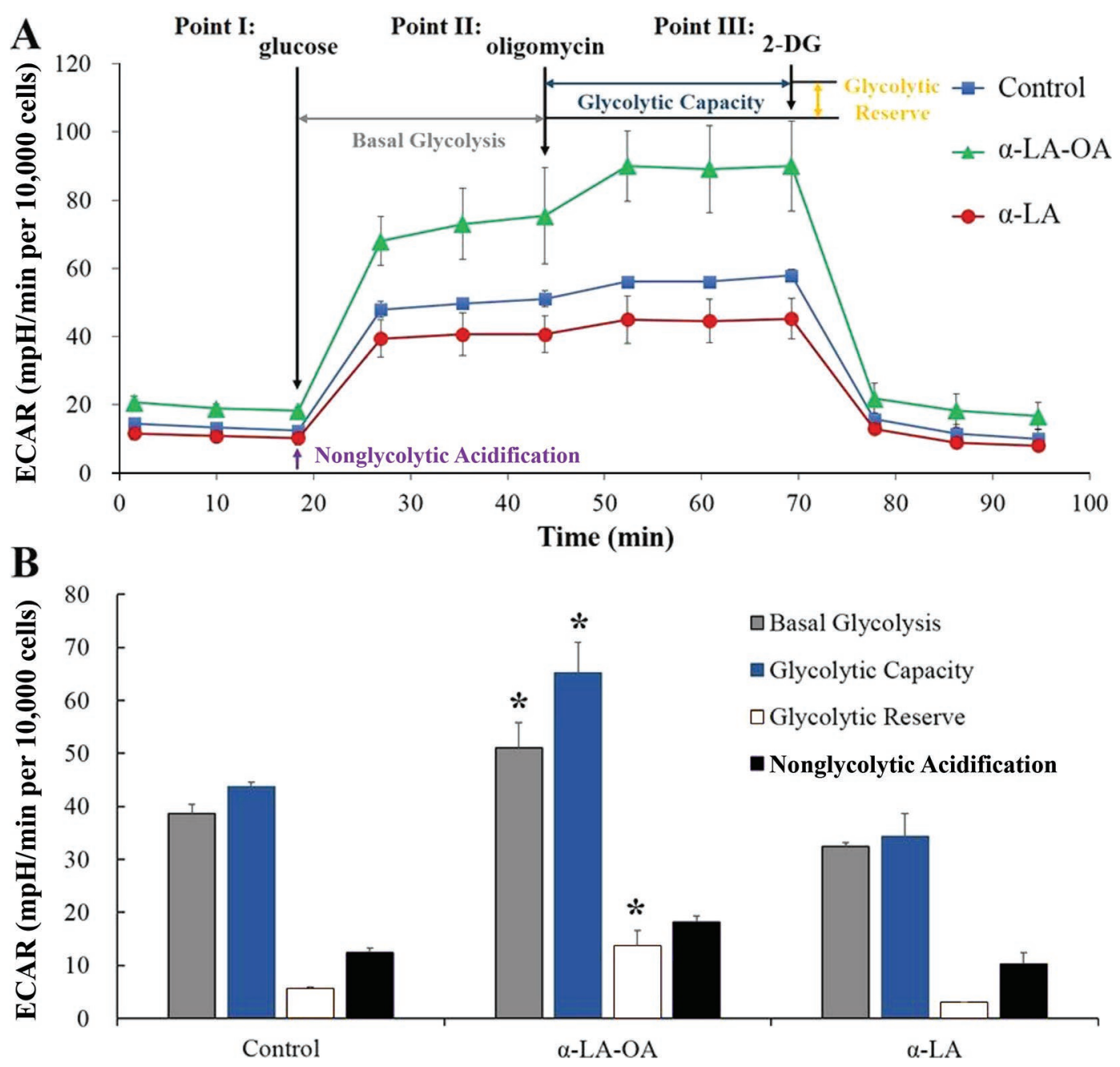

Figure 2. Extracellular acidification rate (ECAR) of HeLa cells after treatment with $\alpha$-lactalbumin-oleic acid complex ( $\alpha$-LA-OA) or $\alpha$-LA for $24 \mathrm{~h}$ in response to glucose stimulation. (A) Cells were starved of glucose for $1 \mathrm{~h}$ before initiation of the experiment, and then $10 \mathrm{~m} M$ of glucose, $1 \mu M$ of oligomycin, and $50 \mathrm{mM}$ of 2-deoxyglucose (2-DG) were added at points I, II, and III, respectively. The ECAR was measured at $37^{\circ} \mathrm{C}$ with a 3-min mix, 3-min wait, and 3-min measurement protocol. The first ECAR measurement was recorded after 12-min equilibration, 3-min mix, and 3-min wait (total time $\sim 100 \mathrm{~min}$ ). The ECAR values were normalized relative to the cell counts in each well. (B) The basal glycolysis, glycolytic capacity, glycolytic reserve, and nonglycolytic acidification were calculated as described in Materials and Methods. Data are presented as averages of 3 to 5 measurements and error bars represent SEM. * indicates a significant difference at a CI of $95 \%$. Color version available online.

added (Figure 2A, point II), which inhibits mitochondrial ATP production and shifts the energy production to glycolysis with the subsequent increase in ECAR by producing lactate acid. This change of ECAR reveals the cellular maximum glycolytic capacity. Finally, a glucose analog, 2-DG was added (Figure 2A, point III), which inhibits glycolysis by competitive binding to hexokinase, the enzyme that catalyzes the first step of the glycolytic pathway. As a result, ECAR decreased to the initial level, which confirmed that the ECAR produced in the experiment was due to glycolysis. Figure $2 \mathrm{~B}$ shows that $\alpha-\mathrm{LA}-\mathrm{OA}$ enhanced the glycolysis of HeLa cells, including the basal glycolysis and glycolytic capacity, compared with controls $(P<0.05)$.
Although tumor cells were prone to enter the glycolytic pathway even when sufficient oxygen is available to support mitochondrial oxidative phosphorylation (OXPHOS), their mitochondrial function is intact and a significant fraction of the ATP might still be derived from OXPHOS (Ward and Thompson, 2012). To investigate the relative proportion of glycolytic flux entering the TCA cycle and the mitochondrial electron transfer chain (ETC) in response to $\alpha$-LA-OA, we measured the basal OCR and OCR in response to inhibitors of ETC function using the Seahorse XF24 extracellular flux analyzer (Figure 3). Inhibition of ATP synthase (complex V of ETC) with oligomycin (Figure 3A, point I) was used to determine the proportion of OCR used 
for ATP production at basal conditions. Then, carbonyl cyanide p-trifluoromethoxyphenylhydrazone was added (Figure 3A, point II), which is an uncoupling agent that collapses the proton gradient and disrupts the mitochondrial membrane potential; as a result, oxygen is maximally consumed by complex IV and OCR is increased. Finally, we applied a mix of rotenone, a complex I inhibitor, and antimycin A, a complex III inhibitor (Figure 3A, point III), leading to the shutdown of mitochondrial respiration and decreased OCR to the level produced by nonmitochondrial respiration. As shown by the calculated mitochondrial function values (Figure 3B), $\alpha$-LA-OA enhanced the basal respiration, maximal respiration, ATP production, and spare respiratory capacity compared with controls $(P<0.05)$.

Tumor cells can use mitochondrial enzymes to meet the demand of macromolecular biosynthesis and to ensure effective cellular proliferation (Ward and Thompson, 2012). Tumor cells also demonstrate metabolic heterogeneity with the capacity to use alternative oxidizable substrates such as glutamine and fatty acids (Weinberg et al., 2010; Pike et al., 2011). Taken together, this suggests that a significant proportion of glycolytic flux may be diverted into lactic acid synthesis in cells treated with $\alpha$-LA-OA, and the enhanced mitochondrial OXPHOS may be due to the utilization of another substrate, such as fatty acid and glutamine, instead of glucose. Therefore, we measured the rate of oxidation of each fuel by measuring OCR in the presence or absence of fuel pathway inhibitors (Figure 4).

\section{a-LA-OA Changed the Utilization of Mitochondrial Substrates}

Both glucose and fatty acids can be converted to acetyl-CoA to enter the TCA cycle though pyruvate and palmitate, respectively. The oxidation of glucose can be inhibited by UK5099 though blocking the mitochondrial pyruvate carrier, whereas utilization of long-chain fatty acids can be blocked by etomoxir, an inhibitor of carnitine palmitoyl-transferase $1 \mathrm{~A}$ that is critical for the translocation of fatty acids from the cytosol into mitochondria. The utilization of AA, such as glutamine, occurs though the conversion of glutamate by glutaminase and then to $\alpha$-ketoglutarate, the direct substrate of TCA cycle, and can be inhibited by BPTES.

Initially inhibiting one pathway measures how dependent the cells are on that fuel source to meet the energy demand, while the use of a combination of the other 2 inhibitors measures the cells' capacity of that fuel in meeting the energy demand. We calculated the OCR changes as shown in Figure $4 \mathrm{~A}-4 \mathrm{~F}$ and found that HeLa cells were more dependent on glutamine instead of glucose and long-chain fatty acids. Treatment with $\alpha$-LA-OA did not influence the dependency on the 3 substrates; however, it increased the capacity of using glutamine and fatty acids to meet the energy demand (Figure $4 \mathrm{G}, P<0.05$ ). Therefore, the increased ATP production and mitochondrial respiration may be due to the enhanced ability to use glutamine and long chain fatty acids.

\section{Gene Expression of Enzymes Regulated Energy Metabolism in Response to a-LA-OA}

We next examined the gene expression of enzymes involved in glycolysis, TCA cycle, and ATP synthesis (Figure 5). Hexokinase (HK) catalyzes the irreversible first step of the glycolytic pathway by phosphorylating glucose to glucose-6-phosphate, which is converted to pyruvate by a series of enzymes including phosphofructokinase, fructose-bisphosphate aldolase (aldolase), and pyruvate kinase. Due to the Warburg effect in tumor cells, pyruvate is converted to lactate acid by lactate dehydrogenase (LDH). There are several isoforms of these enzymes, such as hexokinase (HK1-4), pyruvate kinase (PKM1-2), and LDH, and we chose the isoforms mainly expressed in tumor cells, including HK2 (Wolf et al., 2011; Patra et al., 2013; Guo et al., 2015), PKM2 (Mazurek et al., 2005), LDHA, which favors conversion of pyruvate to lactate, and LDHB, which favors conversion of lactate to pyruvate (Koukourakis et al., 2005; Vander Heiden et al., 2009; Mims et al., 2015).

As shown in Figure 5A, after the 24-h treatment of $\alpha$-LA-OA in which the remaining viable tumor cells had an enhanced glycolysis rate (Figure 2B), we detected a low expression of PKM2 and LDHA compared with controls $(P<0.05)$. The decreased expression of PKM2 was consistent with the results that the sensitivity of tumor cells responded to $\alpha-\mathrm{LA}-\mathrm{OA}$ is dependent on cMyc by inhibiting HK and PKM2 activity (Storm et al., 2011). Furthermore, this may also be the reason for the increased sensitivity of tumor cells to $\alpha$-LA-OA in the presence of 2-DG (Storm et al., 2011). Our previous proteomics results suggested a significantly increased expression of PKM protein and LDHA protein, we thought it was a consequence of the enhanced glycolysis in tumor cells treated with $\alpha-\mathrm{LA}-\mathrm{OA}$. The reason of the decreased mRNA expression of PKM2 and LDHA may be related to the negative feedback regulation of tumor cells.

In the TCA cycle, oxaloacetic acid is circulated after being catalyzed by citrate synthase, aconitate hydratase (ACO2), isocitrate dehydrogenase (IDH1 localized in the cytoplasm and peroxisomes and IDH2 


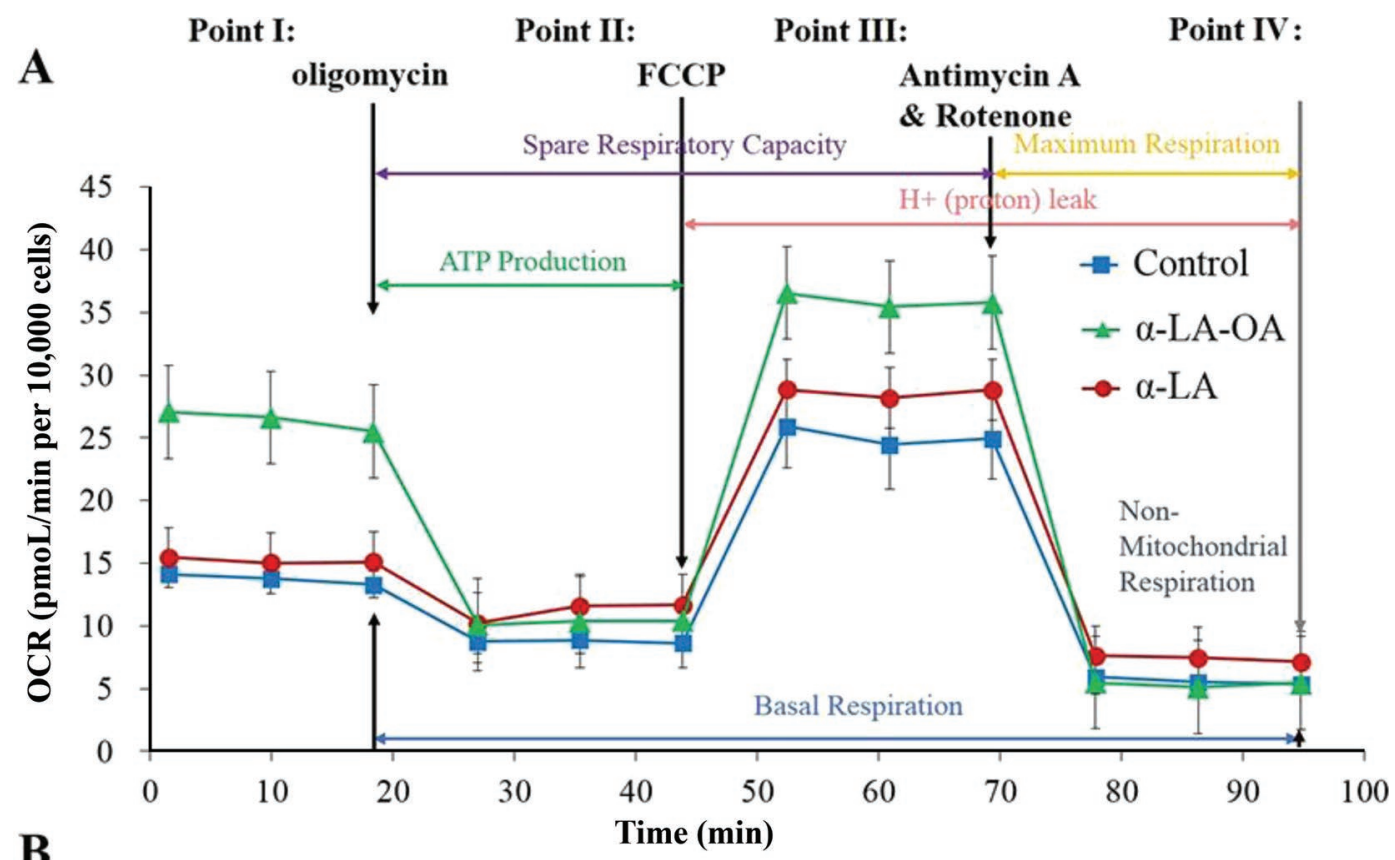

B

Time (min)

Nonmitochondrial Respiration

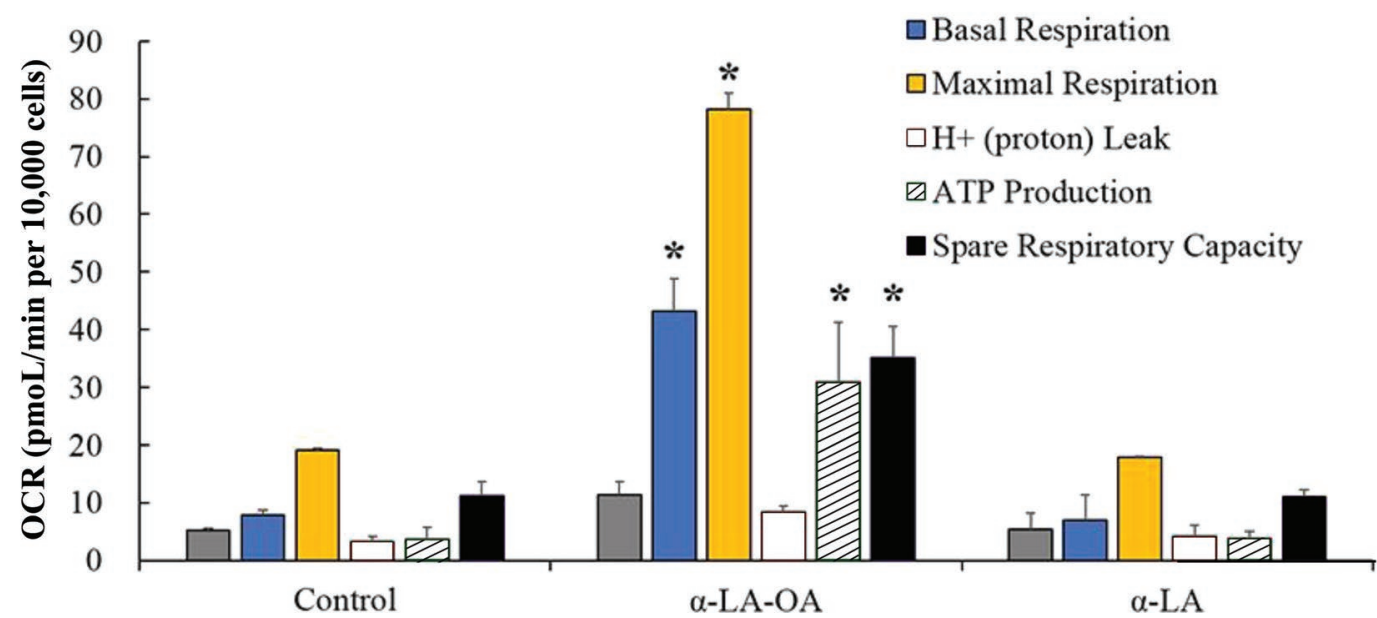

Figure 3. Oxygen consumption rate (OCR) of HeLa cells after treatment with $\alpha$-lactalbumin-oleic acid complex ( $\alpha$-LA-OA) or $\alpha$-LA for 24 $\mathrm{h}$ in response to electron transfer chain inhibitors. (A) Cells were starved of glucose for $1 \mathrm{~h}$ before initiation of the experiment, and then $2 \mu M$ of oligomycin, $1 \mu M$ of carbonyl cyanide p-trifluoromethoxyphenylhydrazone (FCCP), or a mixture of $0.5 \mu M$ of rotenone and $0.5 \mu M$ of antimycin were added at points I, II, and III, respectively. The OCR was measured at $37^{\circ} \mathrm{C}$ with a 3 -min mix, 3-min wait, and 3 -min measurement protocol. The first OCR measurement was recorded after 12-min equilibration, 3-min mix, and 3-min wait (total time $\sim 100$ min). The OCR values were normalized relative to the cell counts in each well. (B) Values of the nonmitochondrial respiration, basal respiration, maximal respiration, proton leak, ATP production, and spare respiratory capacity were calculated as described in Materials and Methods. Data were presented as averages of 3 to 5 measurements and error bars represent SEM. * indicates a significant difference at a CI of $95 \%$. Color version available online.

localized in the mitochondrial matrix), and succinate dehydrogenase A (SDHA). Utilization of long-chain fatty acids requires acetyl-coenzyme A acyltransferase 2 (ACAA2; Cao et al., 2008) and the production of ATP requires electron-transfer flavoprotein $(\alpha$ and $\beta$ subunit, ETFA and ETFB), which acts as an electron acceptor of energy production from AA and fatty acids (Hoffman and Brookes, 2009; Scheonfeld et al., 2010), in addition to the adenosine triphosphate synthase $(\beta$ and $\mathrm{O}$ subunit, ATP5B and ATP5O). As shown in Figure 5B, the gene expressions of $\mathrm{ACO} 2$, which converts citrate to isocitrate, and IDH1, which converts isocitrate to $\alpha$-ketoglutarate (Cairns and Mak, 2013), deceased in response to $\alpha-\mathrm{LA}-\mathrm{OA}$ compared with controls $(P<0.05)$, leaving the reaction of converting oxaloacetic acid to citrate unaffected $(P>0.05)$. Meanwhile, the gene expressions of ACAA2 increased whereas ETFB was inhibited $(P<0.05$; Figure $5 \mathrm{C})$, 
A

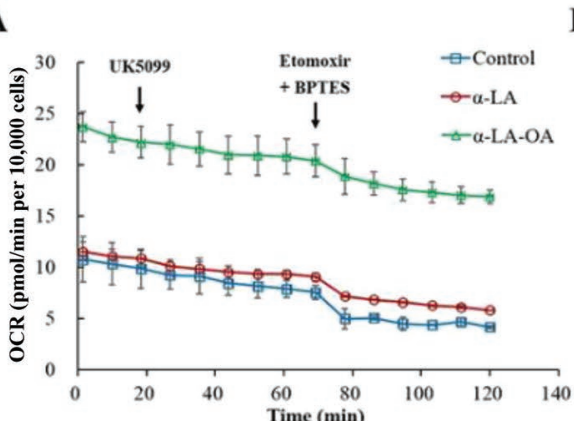

D

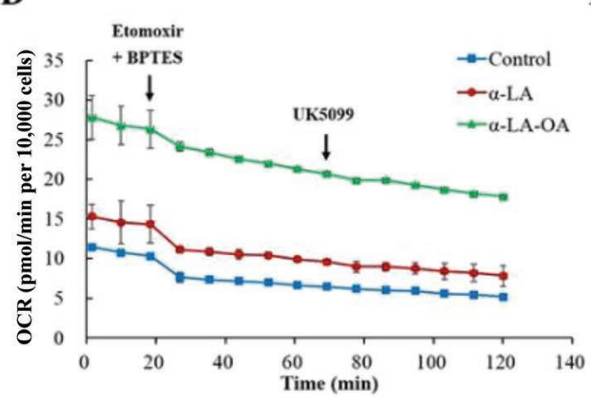

B

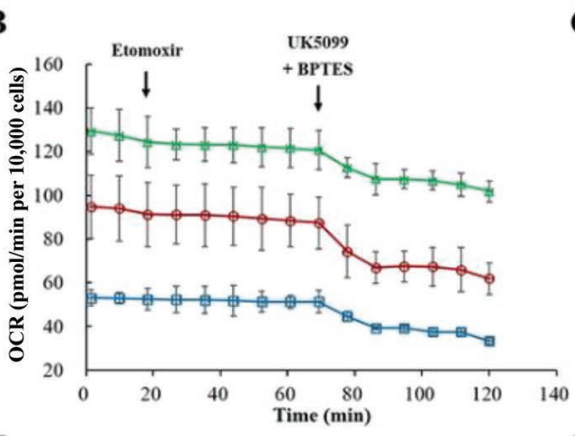

$\mathbf{E}$

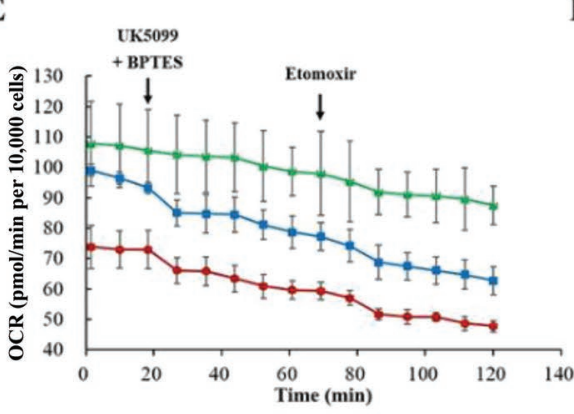

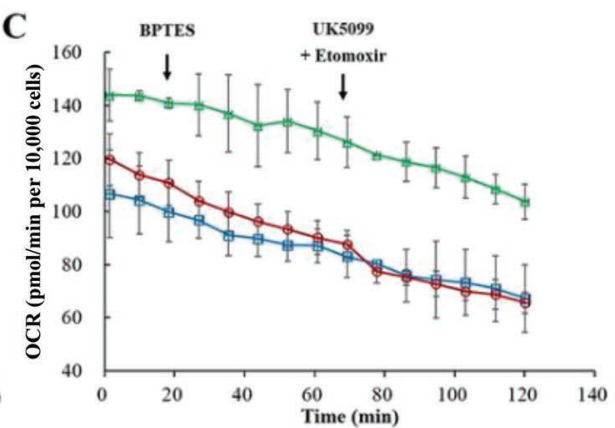

F

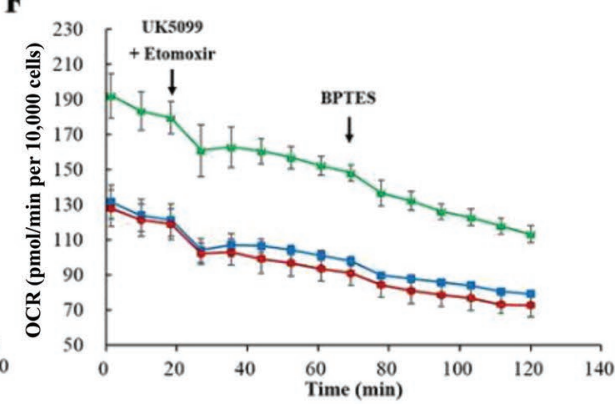

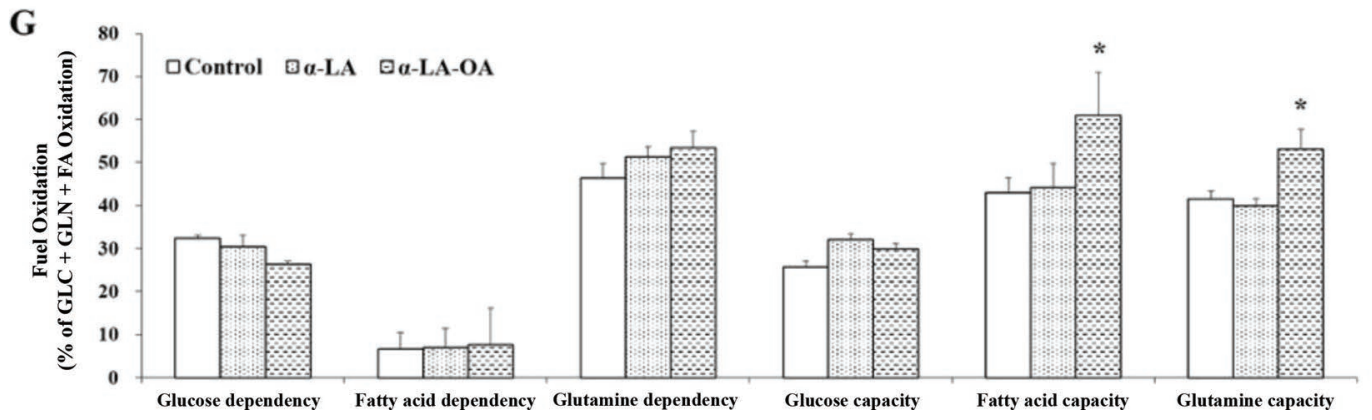

Figure 4. Oxygen consumption rate (OCR) of HeLa cells after treatment with $\alpha$-lactalbumin-oleic acid complex ( $\alpha$-LA-OA) and $\alpha$-LA for $24 \mathrm{~h}$. To measure the dependency of glucose (A), fatty acid (B), and glutamine (C), cells were first inhibited by specific inhibitors UK5099 (3 $\mu M$ ), etomoxir $(4 \mu M)$, or bis-2-(5-phenylacetamido-1,3,4-thiadiazol-2-yl)ethyl sulfide (BPTES; $2 \mu M$ ), respectively, followed by treatment with a mix of the other 2 inhibitors. To measure the capacity of glucose (D), fatty acid (E), and glutamine (F) in meeting energy demand, cells were first inhibited by a mix of the other 2 inhibitors and then the specific inhibitor (UK5099, etomoxir, or BPTES) using the same concentrations as shown in $\mathrm{A}$ to $\mathrm{C}$. The OCR was measured at $37^{\circ} \mathrm{C}$ with a 3 -min mix, 3-min wait, and 3-min measurement protocol. The first OCR measurement was recorded after 12-min equilibration, 3-min mix, and 3-min wait (total time $120 \mathrm{~min}$ ). The OCR values were normalized relative to the cell counts in each well. (G) The dependency and capacity of each fuel were calculated as described in Materials and Methods. * indicates a significant difference at a CI of $95 \%$. The error bars indicate the SD of 3 of 4 parallel samples. Color version available online.

indicating there were insufficient vehicles to transfer the electrons produced by the enhanced $\beta$-oxidation of fatty acids. These results suggested that there was insufficient enzyme activity and mitochondrial function to support the enhanced energy metabolism in the surviving tumor cells. Interestingly, there were also a negative feedback regulation in TCA cycle, ACO protein was found to increased significantly in our previous study (Fang et al., 2016) while its mRNA expression decreased significantly.

Mitochondria are the major source for energy production as well as one of the most important sources of reactive oxygen species (ROS; Brand, 2010). Augmentation of ROS levels is mainly caused by 2 phenomena: either when ATP production exceeds the energy demands of cells or when respiratory chain complexes are impaired or uncoupled (Khawaja et al., 2008; Hagenbuchner et al., 2012). These studies showed that $\alpha$-LA-OA increased ATP production while the expression of the related enzymes was insufficient, which is consistent with the first phenomenon in ROS overproduction. The increased expression of ACAA2 accompanied by a decreased expression of ETFB (Figure 5C) would result in an electron leak when there is insufficient electron transfer to support the enhanced utilization of fatty acids. However, $\alpha$-LA-OA did not influence the proton leak (Figure 3B), indicating that electrons may be used to produce ROS. Furthermore, the reaction catalyzed by 

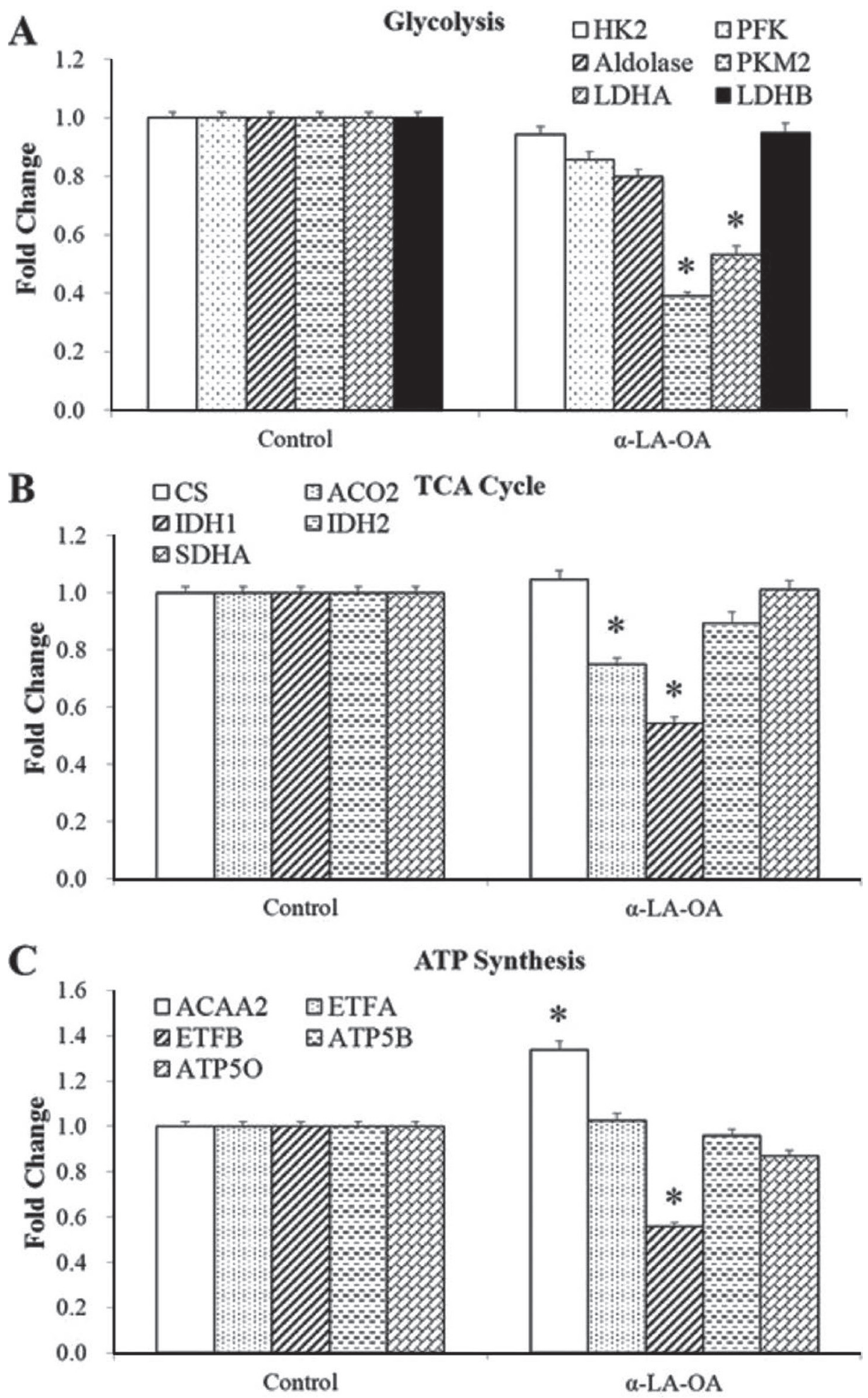

Figure 5. Gene expression of enzymes involved in glycolysis (A), tricarboxylic acid (TCA) cycle (B), and ATP synthesis (C) in response to $\alpha$-lactalbumin-oleic acid complex $(\alpha$-LA-OA) and control. Data with error bars are expressed as mean \pm SD $(n=6)$ * indicates a significant difference at a CI of 95\%. HK2 = hexokinase 2; LDHA = lactate dehydrogenase A; PFK = phosphofructokinase; PKM2 = pyruvate kinase 2; $\mathrm{LDHB}=$ lactate dehydrogenase B; CS = citrate synthase; IDH1 = isocitrate dehydrogenase localized in the cytoplasm; SDHA = succinate dehydrogenase $\mathrm{A} ; \mathrm{ACO} 2=$ aconitate hydratase; IDH2 = isocitrate dehydrogenase localized in the mitochondrial matrix; ACAA2 = acetyl-coenzyme A acyltransferase 2; ETFB = electron-transfer flavoprotein subunit $\beta$; ATP5O = adenosine triphosphate synthase O subunit; ETFA $=$ electrontransfer flavoprotein $\alpha$ subunit; and ATP5B = adenosine triphosphate synthase $\beta$ subunit. 
IDH is also considered a major mechanism of NADPH production in mammalian cells (Cairns et al., 2011) and supplies reducing power to key reactions in several macromolecular biosynthetic pathways and to systems that defend against the oxidative stress induced by ROS (Cairns and Mak, 2013). The decrease in the gene expression of IDH1 (Figure 5B) can lead to an insufficient elimination of ROS. Taken together, this suggests that $\alpha$-LA-OA increases the production of ROS though influencing energy metabolism, which may induce loss of the mitochondrial membrane potential and, as a consequence, activate apoptosis (Circu and Aw, 2010). Previous studies showed that $\alpha$-LA-OA could decrease the membrane potential of mitochondria (Köhler et al., 2001), leading to apoptosis (Gustafsson et al., 2005; Hallgren et al., 2006), and our previous study also found that $\alpha-\mathrm{LA}-\mathrm{OA}$ caused activation of JNK and caspase 9 (Supplemental Figure S1; https://doi.org/10.3168/jds .2017-13731), the mitochondrial-dependent apoptosis pathway. Supplemental Figure S1 shows the expression of the proteins involved in the mitochondrial-dependent apoptosis pathway in response to 40 and $80 \mu M \alpha$-LAOA after $24 \mathrm{~h}$ of treatment.

\section{CONCLUSIONS}

At a concentration of $40 \mu M$ that killed $20 \%$ of HeLa cells, $\alpha$-LA-OA enhanced the total glucose uptake of HeLa cells by increasing the glycolysis potential, mitochondrial OXPHOS functions, and the capacity of utilizing glutamine and fatty acids. However, after 24-h treatment of $\alpha-\mathrm{LA}-\mathrm{OA}$, the gene expressions of PKM2 and LDHA, key enzymes involved in glycolysis, and $\mathrm{ACO} 2$ and IDH1, which regulate the TCA cycle, are decreased, indicating an insufficient ability to support the enhanced glycolysis and OXPHOS in the remaining viable cells. Furthermore, the gene expression of ETFB, which acts as an electron acceptor of energy production from AA and fatty acids, was inhibited. The gene expression of reduced NADPH, which defends against the oxidative stress induced by ROS, was also inhibited because of the decreased expression of IDH1. In conclusion, our results suggest that $\alpha-\mathrm{LA}-\mathrm{OA}$ treatment may induce a condition in which the ATP production exceeds the energy demand, which would lead to the augmentation of ROS and activation of apoptosis.

\section{ACKNOWLEDGMENTS}

This work was financially supported by the National Natural Science Foundation of China (No. 31601443) and the Beijing Municipal Education Commission (China).

\section{REFERENCES}

Andrabi, S. A., G. K. Umanah, C. Chang, D. A. Stevens, S. S. Karuppagounder, J. P. Gagné, G. G. Poirier, V. L. Dawson, and T. M. Dawson. 2014. Poly(ADP-ribose) polymerase-dependent energy depletion occurs through inhibition of glycolysis. Proc. Natl. Acad. Sci. USA 111:10209-10214.

Brand, M. D. 2010. The sites and topology of mitochondrial superoxide production. Exp. Gerontol. 45:466-472.

Cairns, R., I. Harris, and T. Mak. 2011. Regulation of cancer cell metabolism. Nat. Rev. Cancer 11:85-95.

Cairns, R. A., and T. W. Mak. 2013. Oncogenic isocitrate dehydrogenase mutations: Mechanisms, models, and clinical opportunities. Cancer Discov. 3:730-741.

Cao, W., N. Liu, S. Tang, L. Bao, L. Shen, H. Yuan, X. Zhao, and H. Lu. 2008. Acetyl-Coenzyme A acyltransferase 2 attenuates the apoptotic effects of BNIP3 in two human cell lines. Biochim. Biophys. Acta 1780:873-880.

Circu, M. L., and T. Y. Aw. 2010. Reactive oxygen species, cellular redox systems, and apoptosis. Free Radic. Biol. Med. 48:749-762.

Fang, B., M. Zhang, M. Tian, L. Jiang, and F. Z. Ren. 2014. Bovine lactoferrin binds oleic acid to form an anti-tumor complex similar to HAMLET. Biochim. Biophys. Acta 1841:535-543.

Fang, B., M. Zhang, X. Fan, and F. Z. Ren. 2016. The targeted proteins in tumor cells treated with the $\alpha$-lactalbumin-oleic acid complex examined by descriptive and quantitative liquid chromatography-tandem mass spectrometry. J. Dairy Sci. 99:5991-6004.

Fischer, W., L. Gustafsson, A. K. Mossberg, J. Gronli, S. Mork, R. Bjerkvig, and C. Svanborg. 2004. Human alpha lactalbumin made lethal to tumor cells (HAMLET) kills human glioblastoma cells in brain xenografts by an apoptosis-like mechanism and prolongs survival. Cancer Res. 64:2105-2112.

Guo, W., Z. Qiu, Z. Wang, Q. Wang, N. Tan, T. Chen, Z. Chen, S. Huang, J. Gu, J. Li, M. Yao, Y. Zhao, and X. He. 2015. MiR$199 a-5 p$ is negatively associated with malignancies and regulates glycolysis and lactate production by targeting hexokinase 2 in liver cancer. Hepatology 62:1132-1144.

Gustafsson, L., O. Hallgren, A. K. Mossberg, J. Pettersson, W. Fischer, A. Aronsson, and C. Svanborg. 2005. HAMLET kills tumor cells by apoptosis: structure, cellular mechanisms, and therapy. J. Nutr. 135:1299-1303.

Gustafsson, L., I. Leijonhufvud, A. Aronsson, A. K. Mossberg, and C. Svanborg. 2004. Treatment of skin papillomas with topical alphalactalbumin-oleic acid. N. Engl. J. Med. 350:2663-2672.

Hagenbuchner, J., A. Kuznetsov, M. Hermann, B. Hausott, P. Obexer, and M. J. Ausserlechner. 2012. FOXO3-induced reactive oxygen species are regulated by BCL2L11 (Bim) and SESN3. J. Cell Sci. 125:1191-1203.

Hallgren, O., L. Gustafsson, H. Irjala, G. Selivanova, S. Orrenius, and C. Svanborg. 2006. HAMLET triggers apoptosis but tumor cell death is independent of caspases, Bcl-2 and p53. Apoptosis $11: 221-233$.

Hoffman, D. L., and P. S. Brookes. 2009. Oxygen sensitivity of mitochondrial reactive oxygen species generation depends on metabolic conditions. J. Biol. Chem. 284:16236-16245.

Hsu, P. P., and D. M. Sabatini. 2008. Cancer cell metabolism: Warburg and beyond. Cell 134:703-707.

Jiang, Y., L. Lu, Y. Hu, Q. Li, C. An, X. Yu, L. Shu, A. Chen, C. Niu, L. Zhou, and Z. Yang. 2016. Resistin induces hypertension and insulin resistance in mice via a TLR4-dependent pathway. Sci Rep. 6:22193.

Khawaja, N. R., M. Carrè, H. Kovacic, M. A. Estève, and D. Braguer. 2008. Patupilone-induced apoptosis is mediated by mitochondrial reactive oxygen species through Bim relocalization to mitochondria. Mol. Pharmacol. 74:1072-1083.

Köhler, C., V. Gogvadze, A. Håkansson, C. Svanborg, S. Orrenius, and B. Zhivotovsky. 2001. A folding variant of human $\alpha$-lactalbumin induces mitochondrial permeability transition in isolated mitochondria. Eur. J. Biochem. 268:186-191.

Koukourakis, M. I., A. Giatromanolaki, C. Simopoulos, A. Polychronidis, and E. Sivridis. 2005. Lactate dehydrogenase 5 (LDH5) relates 
to up-regulated hypoxia inducible factor pathway and metastasis in colorectal cancer. Clin. Exp. Metastasis 22:25-30.

Mazurek, S., C. B. Boschek, F. Hugo, and E. Eigenbrodt. 2005. Pyruvate kinase type M2 and its role in tumor growth and spreading. Semin. Cancer Biol. 15:300-308.

Mims, J., N. Bansal, M. S. Bharadwaj, X. Chen, A. J. Molina, A. W. Tsang, and C. M. Furdui. 2015. Energy metabolism in a matched model of radiation resistance for head and neck squamous cell cancer. Radiat. Res. 183:291-304.

Mossberg, A. K., Y. Hou, M. Svensson, B. Holmqvist, and C. Svanborg. 2010. HAMLET treatment delays bladder cancer development. J. Urol. 183:1590-1597.

Mossberg, A. K., B. Wullt, L. Gustafsson, W. Mansson, E. Ljunggren, and C. Svanborg. 2007. Bladder cancers respond to intravesical instillation of HAMLET (human alpha-lactalbumin made lethal to tumor cells). Int. J. Cancer 121:1352-1359.

Patra, K. C., Q. Wang, P. T. Bhaskar, L. Miller, Z. Wang, W. Wheaton, N. Chandel, M. Laakso, W. J. Muller, E. L. Allen, A. K. Jha, G. A. Smolen, M. F. Clasquin, R. B. Robey, and N. Hay. 2013. Hexokinase 2 is required for tumor initiation and maintenance and its systemic deletion is therapeutic in mouse models of cancer. Cancer Cell 24:213-228.

Pike, L. S., A. L. Smift, N. J. Croteau, D. A. Ferrick, and M. Wu. 2011. Inhibition of fatty acid oxidation by etomoxir impairs NADPH production and increases reactive oxygen species resulting in ATP depletion and cell death in human glioblastoma cells. Biochim. Biophys. Acta 1807:726-734.
Scheonfeld, P., M. R. Wieckowski, M. Lebiedzinska, and L. Wojtczak. 2010. Mitochondrial fatty acid oxidation and oxidative stress: Lack of reverse electron transfer-associated production of reactive oxygen species. Biochim. Biophys. Acta 1797:929-938.

Storm, P., S. Aits, M. K. Puthia, A. Urbano, T. Northen, S. Powers, B. Bowen, Y. Chao, W. Reindl, D. Y. Lee, N. L. Sullivan, J. Zhang, M. Trulsson, H. Yang, J. D. Watson, and C. Svanborg. 2011. Conserved features of cancer cells define their sensitivity of HAMLETinduced death; c-Myc and glycolysis. Oncogene 30:4765-4779.

Vander Heiden, M. G., L. C. Cantley, and C. B. Thompson. 2009. Understanding the Warburg effect: The metabolic requirements of cell proliferation. Science 324:1029-1033.

Ward, P. S., and C. B. Thompson. 2012. Metabolic reprogramming: A cancer hallmark even Warburg did not anticipate. Cancer Cell 21:297-308.

Weinberg, F., R. Hamanaka, W. W. Wheaton, S. Weinberg, J. Joseph, M. Lopez, B. Kalyanaraman, G. M. Mutlu, G. R. Budinger, and N. S. Chandel. 2010. Mitochondrial metabolism and ROS generation are essential for Kras-mediated tumorigenicity. Proc. Natl. Acad. Sci. USA 107:8788-8793.

Wolf, A., S. Agnihotri, J. Micallef, J. Mukherjee, N. Sabha, R. Cairns, C. Hawkins, and A. Guha. 2011. Hexokinase 2 is a key mediator of aerobic glycolysis and promotes tumor growth in human glioblastoma multiforme. J. Exp. Med. 208:313-326. 